\title{
LQ-based Solution Algorithms for Discrete-time Optimal Control Problems
}

\author{
I-Kong Fong and Jeng-Chung Liu \\ Department of Electrical Engineering \\ National Taiwan University \\ Taipei, Taiwan 10617, ROC \\ ikfong@cc.ee.ntu.edu.tw
}

\begin{abstract}
This paper considers optimal control problems of a class of nonlinear discrete-time systems with respect to cost functions that are additive in the time variable. For problems without state and input constraints, a solution algorith$m$ based on the differential dynamic programming and LQ optimal control techniques is proposed. For problems with constraints, the augmented Lagrangian method is combined with the first algorithm to form a non-primal solution algorithm. Finally, a hydroelectric generation scheduling problem is shown to fit the problem formulation, and is solved by using the algorithms developed.
\end{abstract}

\section{Introduction}

Optimal control methods are important in control engineering. If a type of optimal control problem has a solution algorithm, then control engineers can focus on formulating practical problems in the form required, and use the algorithm to get the optimal control law systematically. This enables control engineers to "weigh" various factors of the problem, and see the effects on the solutions. Thus it is important to develop efficient and easy-to-use solution algorithms for optimal control problems with formulations as general as possible. Unfortunately unless the problem has very special structure, such as the LQ optimal control problem, closed-form solution usually does not exist, and numerical methods that are iterative in nature must be adopted. In this regard, for unconstrained problems having no constraints on states and inputs except the system dynamics, the differential dynamic programming (DDP) technique [1] is a successful example. For the constrained problem, the primal approach of the DDP technique requires that all constraints be satisfied in the iteration process, which results in many quadratic programming problems to solve, and significant increase in computation time.

Here we propose two solution algorithms for the optimal control problems of a class of nonlinear discrete-time systems with respect to cost functions that are additive in the time variable. For unconstrained problems, the algorithm is based on the DDP and LQ optimal control techniques, and is conceptually more straightforward. For the con-

${ }^{1}$ This research was supported by the National Science Council of the Republic of China under Grant NSC 80-0404-E002-48 strained problem, the augmented Lagrangian method is combined with the first algorithm to form a non-primal solution algorithm, which needs not solve any quadratic programming problems. Finally, we show that a hydroelectric generation scheduling problem fits the discussed optimal control problem formulation, and can be solved by using the proposed algorithms.

\section{Main Results}

First, consider the discrete-time optimal control problem

$$
\min _{u_{k}}\left\{J_{N}\left(x_{N}\right)+\sum_{k=1}^{N-1} J_{k}\left(x_{k}, u_{k}\right)\right\}
$$

subject to the system dynamics

$$
x_{k+1}=f_{k}\left(x_{k}, u_{k}\right), \quad k=1,2, \ldots, N-1,
$$

where the initial state $x_{1}$ is known. It is assumed that $f_{k}(\cdot, \cdot)$ and cost functions $J_{N}(\cdot), J_{k}(\cdot, \cdot), k=1, \ldots, N-$ 1 , have continuous first and second order derivatives, respectively. Note that this problem formulation is quite general, except that (1) has the special additive form. For this problem, a solution algorithm based on the DDP and LQ optimal control techniques can be used:

1. Set iteration index $i=0$ and select a sequence of estimated control inputs $u_{k}^{(i)}, k=1,2, \ldots, N-1$.

2. From the estimated control inputs $\left\{u_{k}^{(i)}\right\}$, initial state $x_{1}$, and system dynamics (2), determine corresponding state trajectory $x_{k}^{(i)}$ for $k=1,2, \ldots, N$.

3. Let $u_{k}=u_{k}^{(i)}+\Delta u_{k}$ and $x_{k}=x_{k}^{(i)}+\Delta x_{k}$. Take quadratic approximation of (1) and linear approximation of (2) along $\left\{x_{k}^{(i)}\right\}$ and $\left\{u_{k}^{(i)}\right\}$ to form an optimal control problem in terms of $\Delta x_{k}$ and $\Delta u_{k}$ :

$$
\min _{\Delta u_{k}}\left\{\tilde{J}_{N}\left(\Delta x_{N}\right)+\sum_{k=1}^{N-1} \tilde{J}_{k}\left(\Delta x_{k}, \Delta u_{k}\right)\right\}
$$

subject to the linearized system dynamics

$$
\Delta x_{k+1}=\frac{\partial f_{k}}{\partial x_{k}^{T}} \Delta x_{k}+\frac{\partial f_{k}}{\partial u_{k}^{T}} \Delta u_{k}
$$

where

$\tilde{J}_{N}\left(\Delta x_{N}\right)=\frac{\partial J_{V}}{\partial x_{N}^{T}} \Delta x_{N}+\frac{1}{2}\left[\Delta x_{N}\right]^{T} \frac{\partial^{2} J_{N}}{\partial x_{N} \partial x_{N}^{T}} \Delta x_{N}$, 
$\tilde{J}_{k}\left(\Delta x_{k}, \Delta u_{k}\right)$ has similar but longer expression, $\Delta x_{1}=0$, and all partial derivatives are evaluated at $x_{k}=x_{k}^{(i)}, u_{k}=u_{k}^{(i)}$.

4. Choose appropriate $x_{\Delta k}, u_{\Delta k}$ such that with the variable change $\Delta x_{k}=x_{\Delta k}+\hat{x}_{k}, \Delta u_{k}=u_{\Delta k}+$ $\hat{u}_{k}(3),(4)$ are reformulated into the LQ problem

$$
\min _{\hat{u}_{k}}\left\{\hat{x}_{N}^{T} Q_{N} \hat{x}_{N}+\sum_{k=1}^{N-1}\left(\hat{x}_{k}^{T} Q_{k} \hat{x}_{k}+\hat{x}_{k}^{T} S_{k} \hat{u}_{k}+\hat{u}_{k}^{T} R_{k} \hat{u}_{k}\right)\right\}
$$

subject to the linearized system dynamics

$$
\hat{x}_{k+1}=A_{k} \hat{x}_{k}+B_{k} \hat{u}_{k}+C_{k},
$$

where $Q_{k}, S_{k}, R_{k}, A_{k}, B_{k}, C_{k}$, and $\hat{x}_{1}$ are known.

5. Solve the above $L Q$ problem to get the optimal $\left\{\hat{u}_{k}^{*}\right\}$. Compute $\Delta u_{k}^{*}=u_{\Delta k}+\hat{u}_{k}^{*}$ for all $k$.

6. Update $u_{k}^{(i+1)}=u_{k}^{(i)}+\alpha^{(i)} \Delta u_{k}^{*}$ for all $k$ with some constant $\alpha^{(i)}$ making (1) decrease most.

7. If $\left|\alpha^{(i)}\right| \leq \epsilon$, a prespecified threshold, then stop. Otherwise increase $i$ by 1 and return to step 2 .

In the above algorithm, some matrices need to be nonsingular or positive definite. This indirectly limits the cost functions and system dynamics that can be handled, but it is not too surprising since it is difficult, if not impossible, to develop an algorithm that can handle arbitrary problems. Basically, the above algorithm is similar to the DDP method [1] for the unconstrained problem (1) subject to (2), but conceptually it is more straightforward, as all we need to do are finding quadratic and linear approximations and calling for the standard LQ procedure.

To consider constrained problems, the following constraints are added for $k=1, \ldots, N$ :

$$
h_{k}\left(x_{k}, u_{k}\right)=0 \text { and } g_{k}\left(x_{k}, u_{k}\right) \leq 0 .
$$

Note that each $h_{k}(\cdot, \cdot)$ and $g_{k}(\cdot, \cdot)$ can be vectors. If so, then the inequalities are componentwise. Also, these functions are assumed to have continuous first order derivatives. Unlike the DDP method for constrained problem, which uses the primal approach, we adopt a non-primal approach, the augmented Lagrangian method $[2,3]$. The basic idea is to transform hard constraints into soft ones, i.e., to add into the objective function suitable terms that contain constraint functions, Lagrange multipliers and penalty factors. By adjusting these multipliers and penalty factors systematically and solving the corresponding unconstrained minimization problem with respect to the modified objective function, the solutions will converge to the constrained optimum under mild assumptions. Thus, the above algorithm can be embedded in the lower level of a two-level iterative scheme, of which the upper level is a maximization process updating multipliers and penalty factors to form unconstrained minimization problems for the lower level.

Many practical application problems [4] can be formulated in the form of (1), (2) and (7), and can be solved by the proposed method. Consider the exemplary hydroelectric generation system shown in Fig. 1, which has three reservoirs, two pumped-storage units, and one hydro unit. Let $x_{k_{i}}$ denote the water storage volume of reservoir $i$ at the beginning of hour $k, u_{k_{i}}$ the water released from reservoir $i$ for generation in hour $k, r_{k}$ the volume of natural inflow to reservoir 1 in hour $k$, and $l_{k}$ the system load at hour $k$. Then from water balance relation we have the system dynamics:

$$
\begin{aligned}
& x_{k+1_{1}}=x_{k_{1}}-u_{k_{1}}-u_{k_{2}}-u_{k_{3}}+r_{k} \\
& x_{k+1_{2}}=x_{k_{2}}+u_{k_{1}} \\
& x_{k+1_{3}}=x_{k_{3}}+u_{k_{2}} .
\end{aligned}
$$

Of course, to reflect practical situations, we may have constraints like $\alpha_{i} \leq x_{k_{i}} \leq \beta_{i}$ to represent reservoir storage capacity, $\delta_{i} \leq u_{k_{i}} \leq \gamma_{i}$ to represent bounds on water releases, and $x_{N_{i}}=\kappa_{i}$ to represent final conditions. Note that negative values of $u_{k_{i}}$ 's represent pumping operation. For a given sequence of $l_{k}$, we may want to minimize a cost function of the form:

$$
\sum_{k=1}^{N}\left\{c_{t}\left(l_{k}-\sum_{i=1}^{3} c_{i} u_{k_{i}}\right)^{2}+c_{h} \sum_{i=1}^{3} u_{k_{i}}^{2}\right\}
$$

where the first term stands for thermal generation cost, and the second term stands for lightly weighted hydro generation cost. We implement the above algorithm with the MATLAB and run many cases of the above problem on a TITAN workstation. Different load patterns (e.g., light and heavy loads) and time span (up to 3 days, $N=$ $73)$ are tested. For all cases we obtain convergent optimal control inputs, with about 21 minu tes of CPU time for the most difficult and longest case.

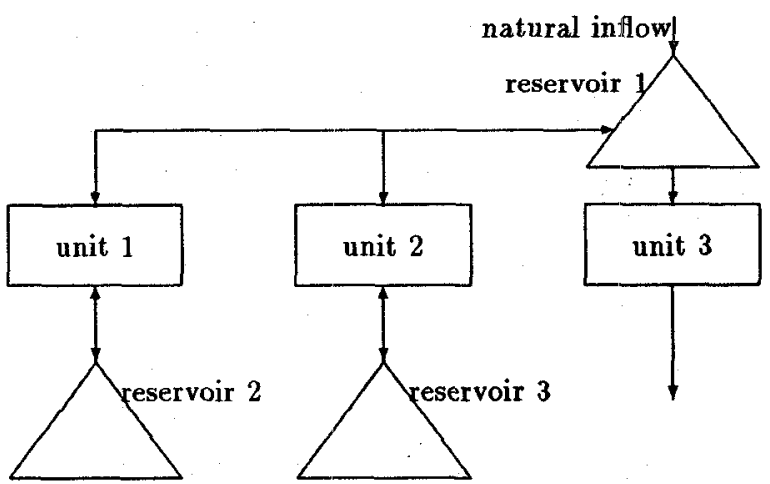

Fig. 1 A hydroelectric generation system.

\section{Conclusion}

We propose an easy-to-implement method for constrained optimal control problems, and apply it to a representative hydroelectric generation scheduling problem. It is believed that this method has the potential for more applications.

\section{References}

[1] D. Jacobson and D. Mayne, Differential Dynamic Programming, New York: Elesvier, 1970.

[2] D. P. Bertsekas, Constrained Optimization and Lagrange Multiplier Methods, New York: Academic Press, 1982.

[3] C. H. Chen, S. C. Chang, and I K. Fong, “An effective differential dynamic programming algorithm for constrained optimal control problem," Proc. American Contr. Conf., Pittsburgh, U. S. A., pp. 1763-1764, 1989. [4] S. C. Chang, C. H. Chen, I K. Fong, and P. B. Luh, "Hydroelectric generation scheduling with an effective differential dynamic programming algorithm," IEEE Trans. Power Systems, Vol. 5, No. 3, pp. 737-743, 1990. 